\title{
Intelligent Agents for Human Behavior Modeling as Support to Operations
}

\author{
Agostino G. Bruzzone, Marina Massei, Simonluca Poggi, \\ Christian Bartolucci and Angelo Ferrando
}

\begin{abstract}
Goal of the present paper is providing support to operations planning and management in complex scenarios. The authors are mainly focused on South Asia region, which is subject of experimental analysis by running an Intelligent Agents -driven HLA Federation. Simulation of investments and operations over an asymmetric mission environment with several parties, insurgents, terrorists and dynamic social framework is the aim. The scenario has various degrees of freedom and $M \& S$ enables evaluation of human behavior evolution and socio-psychological aspects. The presented models include Computer Generated Forces (CGF) driven by Intelligent Agents (IAs) that represents not only units on the battlefield, but also people and interest groups (i.e. Middle Class, Nomads, Clans). The study is focused on Civil Military Co-operations (CIMIC) and Psychological Operations (PSYOPs). The simulation is based on specific architecture that involves various federates playing different roles. Verification, Validation and Accreditation (VV\&A) has been applied along the whole life cycle of the research, in order to determine the correctness and effectiveness of the results. The paper proposes experimental results obtained during the dynamic test of the federations.
\end{abstract}

Keywords Intelligent agents - Human behavior modeling - Simulation of operations · Country reconstruction - CIMIC · PSYOPS

A.G. Bruzzone $(\square) \cdot$ M. Massei

Simulation Team, DIME University of Genoa, via Opera Pia 15, 16145 Genoa, Italy e-mail: agostino@itim.unige.it

M. Massei

e-mail: massei@itim.unige.it

S. Poggi · A. Ferrando

MAST Srl, Corso Podestà 8, 16128 Genoa, Italy

e-mail: poggi@mastsrl.eu
A. Ferrando
e-mail: ferrando@mastsrl.eu
C. Bartolucci

Simulation Team, Via Molinero 1, 17100 Savona, Italy

e-mail: christian.bartolucci@ simulationteam.com 


\section{Introduction}

The human factors are a critical element when investments and operations are planned over a region; indeed the impact of population point of view and the interests of related social layers is often affecting effectiveness and efficiency of operations, it could introduce risks and/or provide opportunities; these elements are obviously pretty difficult to be investigated therefore they strongly affect the overall success; normally it is fundamental to identify all the stakeholders and to consider their interests and their attitude, this require to consider for instance economic, religious, ethnic groups as well towns, villages, local leaders as key factor to be consider in planning.

In several geo-political areas it is required to plan new investments and activities devoted to stabilize or normalize the situation respect previous critical conditions (i.e. civil war, insurgency, terrorism, etc.); in these case it is common to develop initiatives devoted to get support of the local population as well as to improve the quality of life from several point of view (i.e. economy, health, civil rights, security, education, etc.); in order to achieve these results Civil Military Co-operations (CIMIC), Information Operations (INFOPS) and Psychological Operations (PSYOPs) need to be prepared and carried out properly [4].

So it is evident the interest in being able to model these activities as well as the dynamic interaction with the population over a specific framework; indeed such interactions could be pretty complex involving many interest groups representing the different social layers of the population as well as their distribution over the terrain; obviously these elements need to be considered even in reference to the existing situation of the area from many point of view: infrastructures (i.e. roads, hospitals, schools), environmental conditions (i.e. weather), specific actions (i.e. strikes, demonstrations, intimidation activities).

The authors currently have developed models for these context by creating a new generation of intelligent agents representing population and interest groups to drive complex simulation related to these mission environments; therefore it is important to state that these models and the previous considerations could be effectively tailored and applied also in relation to civil scenarios where new investment (i.e. industries and infrastructures) have to be plan over a domestic region or a district as well as during promotional campaign in marketing initiatives.

\section{Simulation Objectives}

Therefore the international context in unstable areas introduce a good motivation to investigate these population models (i.e. country reconstruction operations); currently there it is expected that these models could be very useful for evaluating alternative planning considering risk, opportunities, times, resources costs over a complex and stochastic framework, vice versa the predictive capability of these 
simulators is still pretty limited due to the high degree of uncertainty affecting human elements and the high influence of specific spot events [5]. Due to these considerations, the proposed agent-driven simulations are devoted to conduct experimental analysis and decision support by providing reliable estimations and useful risk analysis, but not the of the exact time and location of a new riot; indeed these events are generated usually by an ignition factor that is highly unpredictable (i.e. a single phrase or shot in a specific moment).

Considering the proposed context it is evident that nowadays military mission environments, especially within countries characterized by different cultures, society organization and changeable political situations, require a new approach to tactical and strategic operations which not only appreciates military engagements, but also relationship between civil population, military forces as well as community evolution and interest groups.

The problem of this analysis is that there are not universally accepted simulation models and that the human behavior modifiers (HBM) are very difficult to be represented; in addition it is even necessary to create models of specific operations that are currently not covered by the existing simulators in order to take of CivilMilitary Cooperation, INFOPS, PSYOPS as well as of psychological consequences over population during mission execution; therefore some existing model/simulator is currently taking into account these not-kinetic operations, but usually it is just a qualitative on/off parameters or a manual script affecting the scenario evolution; this obviously don't allow to consider the complex dynamic of the interaction among different interest groups that is the basis for situation evolution.

\section{Application Framework and Proposed Approach}

The simulator should consider for instance that digging a well within an area could generate positive effects on some part of population (i.e. people hired to carried out the work, owner of the land) as well as negative effects on other ones (i.e. opposite clan respect well owner, opposite political party respect that one involved); these actions generated direct impact on element of the population living in the area as well as on the their related interest group and in addition produce a cascade effect on all the social networks among people and interest groups. In addition if due to weather conditions and/or lack of resource the well constructions result to be affected by delays this could produce negative impact on the people that expect the completion to get benefits of this asset.

All these elements as well as the cascade of effects could result positive or negative with a strong influence due to the dynamically evolving relationships among people and interest groups and also due to the importance of the specific actions, the cultural background and the communications [15].

Indeed the diffusion in the region and among the people and interest groups of the effects of the actions is modeled based on communications over different supports (face to face, media, phones) and considering specific factors; therefore 


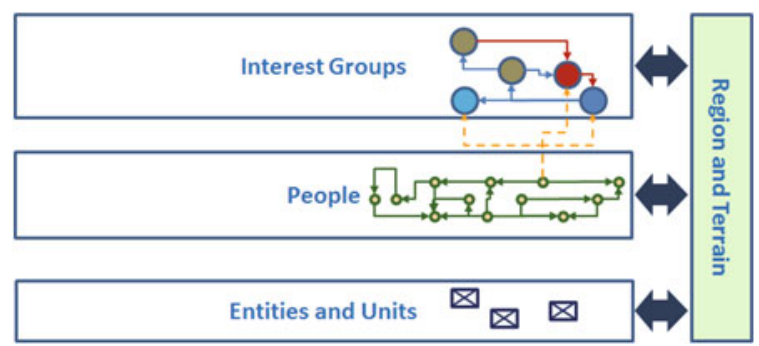

Fig. 1 Multilayer model including population, interest groups and units

these communications introduces attenuation factors and delays; due to the computational workload (i.e. in our case 300,000 people and 60 interest groups) the cascade effect could slow down simulation on single workstations, for this reason it is possible to run the simulation with correct diffusion models or by considering that the diffusion happen with fixed stochastic delays along each single operation phase (this reduces of drastically the events to be considered); considering multiple actions on going concurrently and the main interest to measure final effects this simplification resulted acceptable, therefore if computation power is available it is possible to run the simulator using more correct models.

In the proposed models it was required to model these elements and to create a simulation able to reproduce a scenario where CIMIC and PSYOPS were conducted; the interest group and populations were modeled based on a multilayer approach that consider both population elements deployed over the terrain and interest groups; in addition these entities interact with the units of the different parties (i.e. coalition forces and insurgents).

Due to the fact that the intelligent agents reproduce population behavior within operations, the model allows the users to correctly evaluate the reaction of the civilians not only to military actions, but also during peace keeping and reconstruction phases.

The research has been developed and tested through the involvement of Subject Matter Experts (SME) from different countries; in particular the proposed scenarios was developed as demonstration for a R\&D (Research and Development) project named CIMIC and Planning Research in Complex Operational Realistic Network (CAPRICORN) (Fig. 1).

\section{Intelligent Agents and Simulation Development}

These models have been created in order to support operational planning decisions and to be integrated with other systems previously developed; it is able to work as single user or within a federation architecture (HLA standard) [12]; this was motivated not only by the needs of respecting existing standards, but even for the opportunity provided by developing an open architecture to be further integrated 
with other simulations in order to cover complex problems. Due to these reason interoperability requirements are pretty important and represent a strategic advantage of the proposed approach [1, 2, 4-18]. In fact IAs (Intelligent Agents) [10]; have a big potential in addressing these kind of problems [17].

The most important feature of the models is represented by the intelligent agents that are able to simulate human behavior of people modeling their characteristics; this not only in term of their party side (neutral forces, friends, enemies and civilians), but even in reference to their liaisons to different interest groups and social networks. The capability to use this approach it is an important support for applications involving federations of simulation to address complex scenarios and multiple threats for training and it is pretty interesting to investigate their use for supporting operational planning.

In the past the authors set up libraries of innovative models able to simulate different society attributes represented by riots, agitators and terrorists (RATS) and IA-CGF modules [1, 6]; in some case it was possible to simulate the whole population of a large area reacting to a natural disasters [3] of a big town respect humanitarian activities [7] or in relation to health care issues [9]; therefore in this case the intelligent agents were extended to cover, not only entities and units operating on the field as well as single individuals/families within the population, but even social objects such as interest groups.

In order to succeed in this process it becomes necessary to properly design, tailor and experiment the scenario considering the very large quantity of elements, variables and parameters; due to these reasons the M\&S (Modeling and Simulation) process is formulated over three phases: simulation development, specific mission environment tailoring and simulation experimentation over the specific mission environment. The authors decided to develop an innovative model of a whole country, taking into account the features that involve agents able to correctly interact in the agent based environment; obviously considering the very broad spectrum of applications and elements affecting these operations it is critical to restrict the range of validity and the components to model based on a detailed analysis to be carried out among trans-disciplinary teams involving scientists and operative people [9]. Indeed considering the possibility to use these agents in order to support decision makers on the field for planning operations in overseas scenarios it could be very important to develop a simulator able to be used by people with no strong scientific background and using limited computing capability. Therefore it is necessary to develop models and simulators able to run correctly based on an installation and configuration that should be operated and maintained on field with the kind of resources that are expected to be available on that context; users should be able to configure and create a mission environment; therefore considering the complexity of these applications it is expected that a team of experts and analysts will proceed to create a configuration of the simulator for a specific region and timeframe (i.e. Kapisa District in Afghanistan 2010) to be used as reference by final users eventually deployed on site.

More in detail, the first step approached by the scientists consist of Modeling phenomena, actions and elements that are specific for a socio-political-cultural 
framework. During the conceptual model creation, simulation expert contributions are essential to building a proper and effective set of models and to properly approach the problem thanks to their knowledge of specific operations and scenarios; for instance for a region could emerge the necessity to include nomad behaviors among the possible alternative occupation of the population.

The second phase regards precisely the knowledge management: info sources are used in order to achieve the knowledge basis in order to tailor parameters and entities of a specific region or context; indeed to the necessity to determine a reference scenario a specific mission environment has to be defined; for instance it is necessary to collect information about the different political and economic groups as well as to tailor the importance of religion and clan factors in term of their influence in creating a family respect the specific cultural area. Obviously along the entire M\&S process the VV\&A has been performed to ascertain their formal correctness and their usability effectiveness according to the imagined use; therefore during the simulation experimentation is the critical moment to dynamically test the validity of the models as well as the functionality of the simulator. A set of mission environment hypothesis are defined by planners and/or analysts in order to choose alternative friend course of action (F-COA) and opposite course of action (O-COA); by this approach it is possible to plan investments and operations; a COA could involve CIMIC or PSYOPS targeting different interest groups over a specific zone, affecting people in the area as well as social layers; in addition the decision maker could define the operation time plan of the investment, the assigned resource in term of money as well as equipment and people; obviously during the simulation multiple operations could be planned and carried out concurrently or sequentially and the simulator allow to consider availability of resources, influence of opposite force actions as well as weather condition influence (i.e. weather working days for external constructions).

Each CIMIC or PSYOPS evolves based on different phases (i.e. for a CIMIC planning, engineering, acquisition of resources, erection, commissioning) each one affected by specific needs in term of money, resources, boundary conditions (i.e. weather).

The agents are currently driving the behavior of population and interest groups respect their perception of the general situation and their "feelings" respect ongoing activities; the models use fuzzy rules to estimate the effect of the different operations respect their nature and their attitude respect the actors.

Relationships among entities are usually defined usually by two functions (impact and influence) that could be defined in term of ownership to membership functions respect the following classes (low, medium, high and negative, indifferent and positive) as proposed in the following graph. By this approach it is possible to express quantitative estimations about the effectiveness of the actions conducted; for instance it is possible to apply de-fuzzification in order to transform the relationships among different critical interest groups or people over an area in order to estimate their trustiness respect specific players; for instance the Overall Trustiness of the Population respect the Coalition could be estimated or that one of a specific religious group, village and/or district. For each mission environment the simulation 
Intelligent Agents for Human Behavior Modeling ...

parameters have to be set according to the initial conditions and hypothesis; then predefined settings represent the base for the execution of the simulator, which outputs has to be analyzed (Fig. 2).

The simulation execution could run in different operative modes according to the context and user requirements: stand alone, federated with other simulators, multiple replicated runs etc.; at the end the outputs are collected for each single run and statistically analyzed; these results are evaluated during the simulation experimentation [16]; a classic and simple approach for analyzing the results it is based on what if analysis consists of the simulation of different hypothesis previously formulated. Another possibility that is allowed by simulator is to compare the Desired Final Effect (DFE) of a scenario with the Simulated Course of Action (COA).

The user can also first define Key Performance Indexes (KPIs) and then compare final results of different planning alternatives basing evaluation on them; an effective approach to perform a ranking of different alternatives consists of creating a target function which has to be able to appreciate and involve all these Key Performance Indexes. The latter also represent the reference in order to develop the cost-benefits analysis, which, together with risk analysis, gives to the user all the elements for choosing the best planning alternative. Considering the complexity of the mission environment usually Design of Experiments (DOE) is used in order to complete analysis and produce synthetic reports [11].

Through the feedback from military users with operational experiences and subject matter experts on the specific disciplines, it was possible to develop the models as well as to define the specific user needs; by this approach to develop and validate the conceptual models, to perform the definition of the specific mission environment created for CAPRICORN Demonstrator and to validate the functions; during the last phase related to use the Demonstrator for testing and analyzing simulation results it was possible to complete the dynamic VV\&A of the proposed approach over a specific case study. In particular the involvement of the users for

Fig. 2 Fuzzy membership for evaluating mutual relationships between two groups of interest

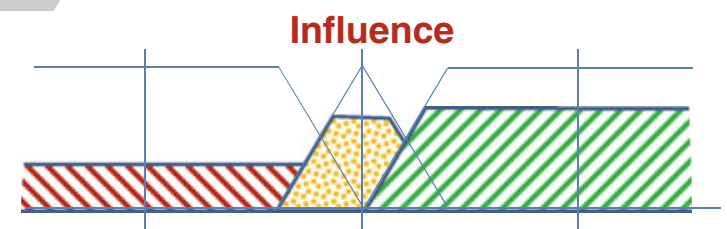

Negative

Indifferent

Positive

Impact

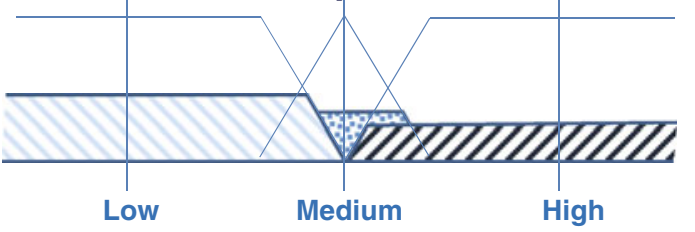


Fig. 3 Simulation interface

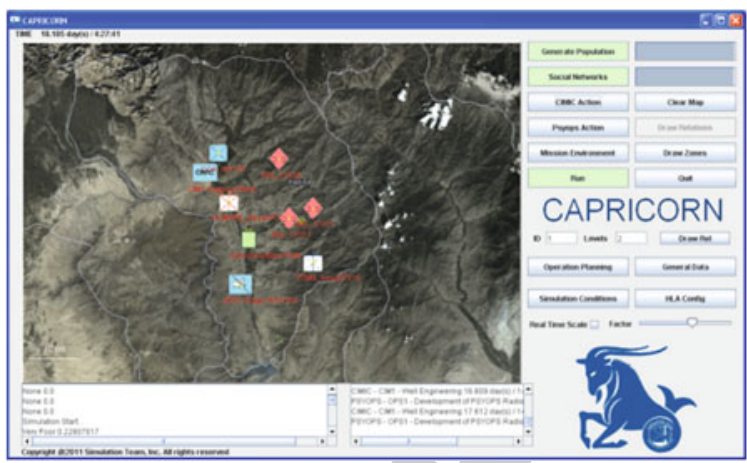

VV\&A was based on different phases; during the first one the focus was to review of key concepts and operational planning requirements; this allowed to generate a common synthesis about CIMIC and PSYOPS, decision making processes, scenario analysis methodologies, training and risk analysis requirements; most of the activities carried out in this phase was organized mostly by desk-top review and face validation performed through organization of meetings and workshops; therefore in this phase some preliminary simplified model was presented and even executed to share concepts and to validate and verify model assumptions and IA basic behaviors. In the following phases the work was based on running of the simulation in front of the users and on analyzing experimental results: during this phase operational planning was carried out through the cooperation of users, analysts, subject matter experts, development team and operational planners (Fig. 3).

\section{Population Model}

For a tactic scenario, such as the real recently warfare where Northern Atlantic countries are involved, is necessary to model civil status and characteristics like:

- Ethnic and Clan

- Religion;

- Cultural/educational level;

- Social and Economic Status

- Geographic location of object;

- Gender;

- Age;

- Health care status;

- Political party;

- Psychological status; 
Table 1 Classes included in the layers

\begin{tabular}{l|l}
\hline Classes & Description \\
\hline Terrain & Infrastructure Layers (i.e. Roads), Terrain Elements, Weather Conditions \\
\hline People & The Population Entities on the Terrain (i.e. Mrs. Baran and/or Sakhi Family) \\
\hline Groups & $\begin{array}{l}\text { Interest Groups (i.e. Sunnis, Honey Producers, Hotaki Clan, Tajik Ethnic Group, } \\
\text { Hamnazar Political Party) }\end{array}$ \\
\hline Entities & Units on the Terrain (i.e. Coalition Platoon, Insurgent Group, Riot) \\
\hline
\end{tabular}

And especially are considered particular modifiers of person features, such as:

- Stress;

- Fear;

- Aggressiveness;

- Fatigue;

- Trustiness.

People relationships, friendships and social relationships are also considered in agents algorithms and distributed in a stochastic manner using Monte Carlo techniques based on consistency algorithms able to aggregate people respect the sociocultural-economic background (Table 1).

People have their social networks interconnecting the population elements based on familiar and friendship relationships; while each people object is connected to multiple interest groups based on his nature with dynamic links representing his affiliation and the related strength; in addition groups and interconnected by mutual hostility and friendship over the social layer, obviously also these connections evolve dynamically during the simulation due the actions carried out; in addition the terrain and Entities affect the behavior of people and social layers, while the Intelligent Agents are in charge of directing the objects during the simulation for completing tasks and for reacting to stimuli and to their own situation awareness.

\section{Demonstrator Results}

The Capricorn Demonstrator consists of a Simulator including the Mission Environment Generator based on Monte Carlo technique applied on statistical database of the population; by this approach the whole people objects representing population and all related interest groups are created and interconnected by the reference relationships over the different layers.

It is proposed an example specific to a CIMIC/PSYOPS mission environment in the Kapisa Afghan region, considering the related COA and parameters concerning population, social networks and groups. The simulation paradigm is based on stochastic discrete-events simulation and it is federated within an HLA Federation (High Level Architecture) both referring to original and IEEE1516 standards; models were implemented in Java with different RTI (Run Time Infrastructures) were tested including Portico, Pitch, VT Mäk. During the test federation integrated 
CAPRICORN Simulator and IA-CGF E\&U (Intelligent Agent Computer Generated Forces Entities and Units) developed by Simulation Team for modeling units on the battlefield; the simulation were carried out over Kapisa Region in South Asia considering presence of several companies of Coalition Forces, several units of Insurgents able to carried out O-COA (i.e. Intimidation), Demonstrations and Riots generated based on the population behaviors and simulated within IA-CGF E\&U; the operations (i.e. CIMIC and PSYOPS) as well as the Interest Groups and Population were simulated by CAPRICORN Demonstrator over a timeframe corresponding to 1 year. ANOVA (Analysis of Variance) was applied in order to measure the confidence band on the controlled variables and the optimal duration time [14]. As anticipated the population and social networks, within the simulation, are generated by CAPRICORN Demonstrator basing on Monte-Carlo techniques; Groups and people so generated relate with entities and units as well as with PSYOPS/CIMIC operations; the actions and events are affected by stochastic factors considering time, cost and effectiveness elements as well as all human behavior factors.

The outputs of CAPRICORN are related to performances in term of times, costs and involved resources during the planned operations as well as a KPI concerning the evolution of the mutual behavior between the critical groups represented in the chosen scenario (i.e. trustiness of the target groups of the operation respect coalition forces, or overall trustiness of the population yersus coalition forces).

A sensitivity analysis based on DOE was carried out respect different independent variables such as:

- A: Budget Allocated to the main Operation

- B: Staff and Resources Assigned to the main Operation

In the Fig. 4 the effect represents the influence expressed as ratio between contrast and the square pure error of trustiness scalar of the target function "overall trustiness"; the analysis propose the effect of single independent variables and of their combination respect this output.

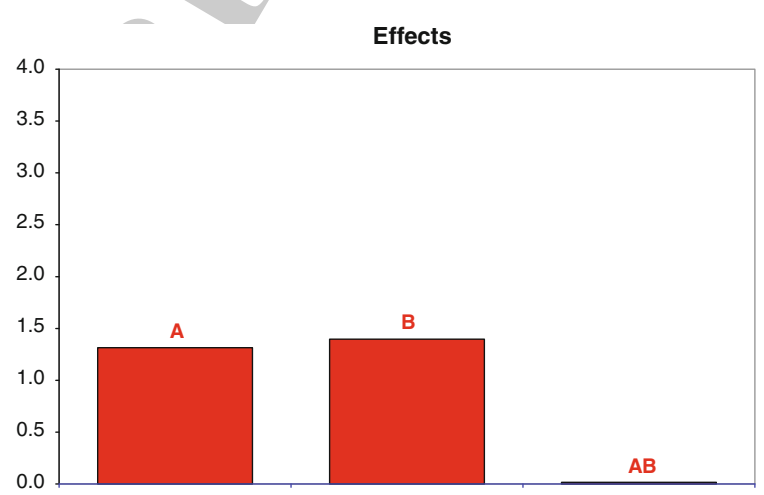

Fig. 4 Estimation of the effect of planner degrees of freedom (budget and resources) on the trustiness between the two critical Groups of Interest (Coalition and Target Group) 
Trustiness COA pro Sunni Digging Well Action

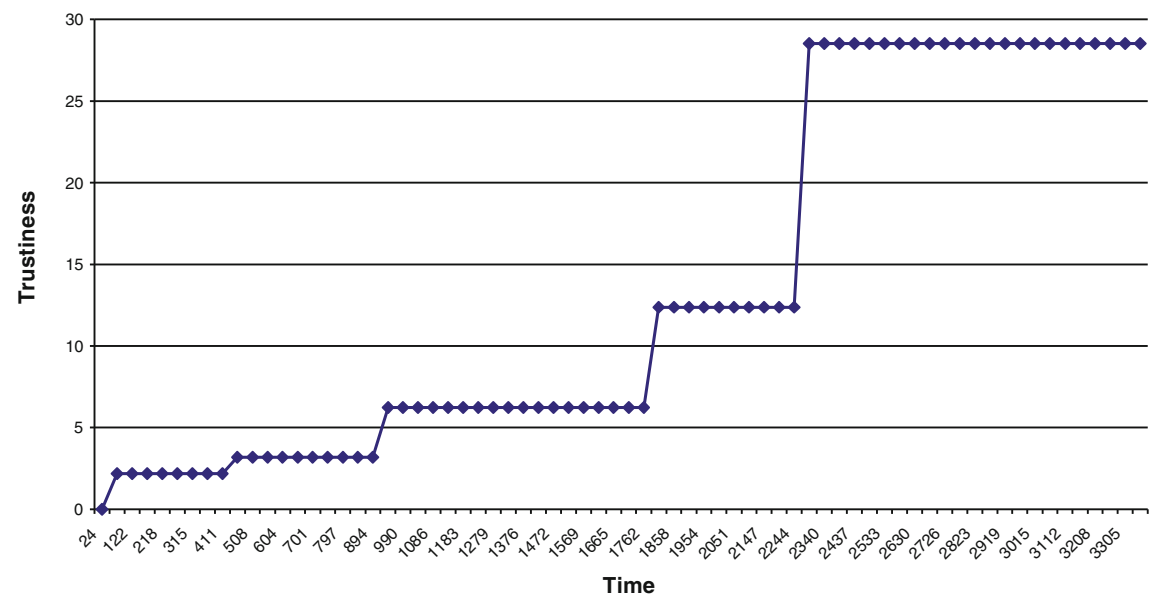

Fig. 5 Trustiness evolution with simplified model

At the end of the simulation process the user knows the effective schedule of the different operation phases (i.e. planning, preparation, supplying, erection/execution, commissioning/follow-up) and the overall duration as well as costs, cash flow, impact on the population.

The figure below shows the evolution of trustiness during the simulation of the CIMIC action well digging COA; it is evident that the deliverables of the different phases introduce major changes; in fact the simulation in this case was executed with the simplified algorithm for diffusing of positive/negative reinforcement due to the action among the population the cascade (Fig. 5).

The model allow simulating multiple CIMIC and PSYOPs actions within different zones and affecting different groups; these could be planned and studied over a single simulation run: in this case report concerns information about their changing along the time and their effect on the population. So for example the solution which determines the best impact on civilians could be identified and quantified as well as risk estimation could be used to support decision making.

The authors provide the experimental analysis carried out through the final reports of CAPRICORN simulation; in effect it is possible to evaluate and test different courses of actions by planning and simulating different CIMIC/PSYOPs in term of type, location, target group, promoting group, duration, budget, resources. Concerning with the experimental analysis, the authors applied Mean Square pure Error and Sensitivity Analysis for different CIMIC \& PSYOP actions by considering a 2-level factorial design respect to the trustiness level as target function.

The figure below represents the mean square pure error diagram for a CIMIC action Digging Well from COA in term of variance of the trustiness respect the 


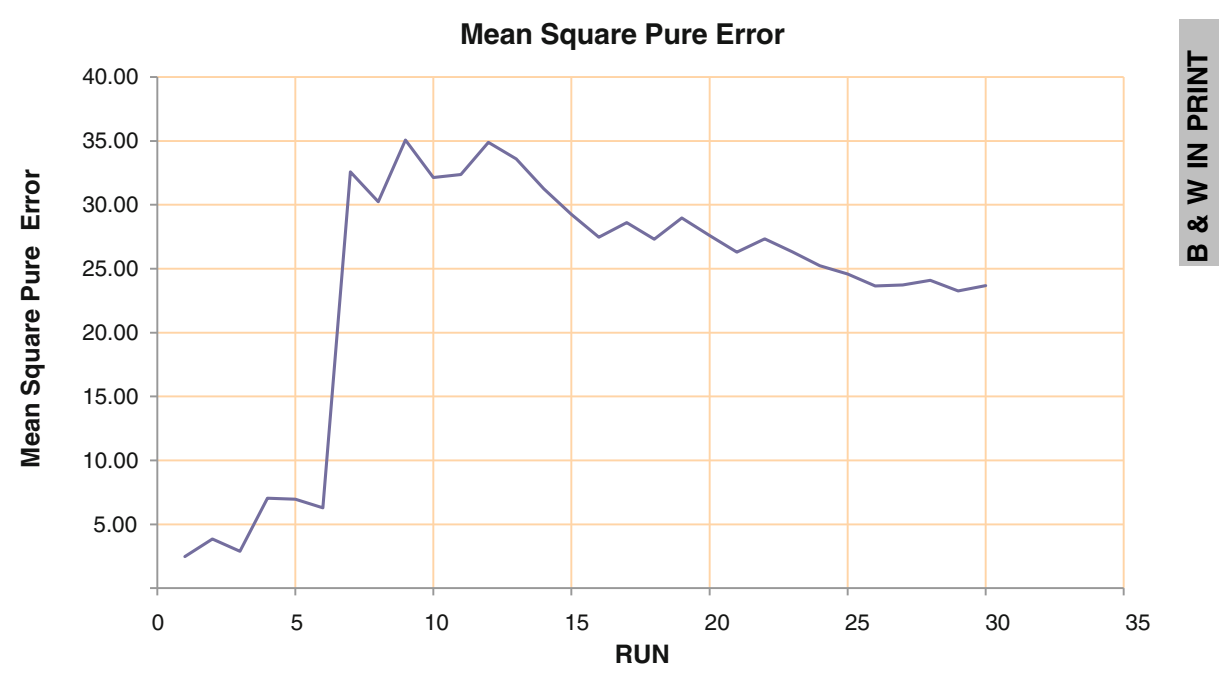

Fig. 6 Mean square pure error diagram for a CIMIC action digging well from COA pro Sunni in Zone 0 (Run expresses the pure number of replications; MspE express the trustiness variance; Trustiness $[-100$ to 100$])$

replicated runs carried out by changing the random seeds of the statistical distributions; it is evident with 25 replications it is possible to obtain results stable with acceptable confidence in term of trustiness $(\sim 15 \%)$. For instance, considering a CIMIC action Digging Well from COA pro Sunni in Zone 0 the MSpE (Mean Square pure Error) was the following:

The Experimental Error is stable between the 26th and the 30th run. So it is possible to state that 26 run are needed for a correct evaluation of the experimental error. By considering the sensitivity analysis related to the independent variables Resources and Budget, both are significant and have positive effects on the population trustiness level (Fig. 6).

In Fig. 7 the contrast represent the influence of factor expressed respect the target function "Trustiness among Coalition and Sunni Interest Group". The model is available to be used for several different kind of investments and infrastructures such as school construction, police station installation and digging wells and it could be extended easily to irrigation infrastructures, buildings and apartment constructions, roads; in addition in term of INFOPS and PSYOPS it is already possible to simulate use of Radio and TV Media as well as leaflet campaign over a region. 


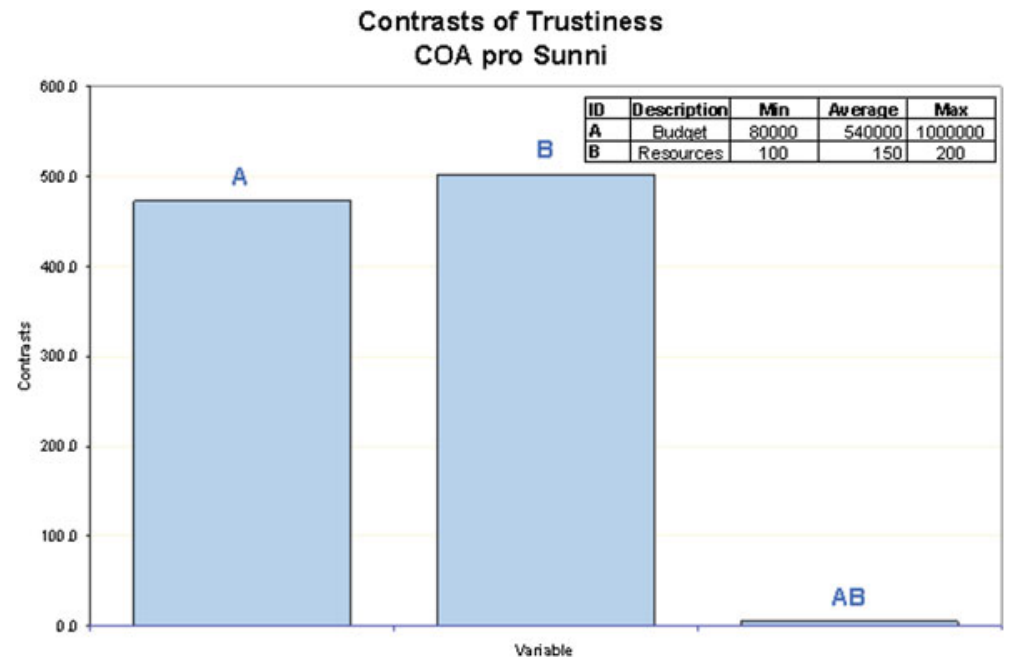

Fig. 7 Contrasts diagram related to trustiness target function respect of budget and resources (Contrast represent the influence of a factor expressed respect trustiness as scalar [-100 to 100]; Budget [Euro] and Resource [people])

\section{Conclusions}

This paper propose an approach to model operations devoted to create infrastructures and actions on a area to improve the social economic situation; the authors developed innovative models for the population and the interest groups devoted to reproduce their behavior and to estimate the impact of the new actions the context is referring to the case of CIMIC, INFOPS and PSYOPS conducted in South Asia and consider both economic and operational aspects as well as weather conditions and possible hostile actions by insurgents.

The research proposed by the authors represents a modeling approach for reproducing complex behavior among population and interest groups during specific operations. The experimental analysis provided interesting results and confirmed the potential of this approach; currently the authors are working for further extend the current models for different applications including industrial and civil cases over domestic scenarios.

Acknowledgments The authors are very glad to thank EDA-European Defence Agency, the Italian MoD (Ministry of Defence) and French MoD that sponsored CAPRICORN Project. For further information please visit www.liophant.org/projects/capricorn. 


\section{References}

1. Bruzzone AG (2008) Intelligent agents for computer generated forces. Invited Speech at Gesi User Workshop, Wien, Italy, Oct 16-17

2. Bruzzone AG (2011) Human behavior modeling as an enabling technology for our world challenges. Invited Speech I3M2011, Rome, Italy, September

3. Bruzzone AG, Massei M, (2006) Modeling for estimating impact on road transportation of regional emergencies \& disasters. In: Proceedings of HMS, Barcelona, Spain, October

4. Bruzzone AG, Massei M (2007) Polyfunctional intelligent operational virtual reality agent: PIOVRA final report, EDA Technical Report

5. Bruzzone AG, Massei M (2010) Intelligent agents for modeling country reconstruction operation. In: Proceedings of AfricaMS 2010, Gaborone, Botswana, Sept 6-8

6. Bruzzone AG, Scavotti A, Massei M, Tremori A (2008) Metamodeling for analyzing scenarios of urban crisis and area stabilization by applying intelligent agents. In: Proceedings of EMSS2008, Sept 17-19, Campora San Giovanni (CS), Italy

7. Bruzzone AG, Massei M, Tarone F, Madeo F (2011) Integrating intelligent agents \& AHP in a complex system simulation. In: Proceedings of the international symposium on the AHP, Sorrento, Italy, June

8. Bruzzone AG, Tremori A, Massei M (2011) Adding smart to the mix. Model Simul Training: Int Defense Training J 3:25-27

9. Bruzzone AG, Novak V, Madeo F (2012) Agent based simulation model for obesity epidemic analysis. In: Proceedings of I3M2012, Wien, Austria, September

10. Calfee SH, Rowe NC (2004) Multi-agent simulation of human behavior in naval air defense. Naval Eng J 116(4):53-64

11. Hill D (1996) Object-oriented simulation. Addison Wesley, Reading

12. Kuhl F, Weatherly R, Dahmann J (1999) Creating computer simulation systems: an introduction to the high level architecture. Prentice Hall, Upper Saddle River

13. Montgomery DC (2000) Design and analysis of experiments. Wiley, New York

14. Mosca R, Giribone P, Bruzzone AG (1993) Optimum search techniques applied to studies relative to plant problems performed by means of simulation. In: Proceedings of SimTec93, November, San Francisco (CA)

15. Ören TI, Yilmaz L (2009) On the synergy of simulation and agents: an innovation paradigm perspective, special issue on agent-directed simulation. Int J Intell Control Syst (IJICS), vol 14, Nb. 1, March 4-19

16. Ören TI, Yilmaz L (2012) Agent-monitored anticipatory multisimulation: a systems engineering approach for threat-management training. In: Proceedings of the 24th European Modeling and Simulation Symposium, September 19-21, Austria, pp 277-282

17. Seck M, Frydman C, Giambiasi N, Oren TI, Ylmaz L (2005) Use of a dynamic personality filter in discrete event simulation of human behavior under stress and fatigue. Found Augemented Cogn 11:908-917

18. Spiegel MR, Schiller LJ (1999) Statistics. McGraw Hill, NYC

19. Yilmaz L, Ören $T$ (2010) Intelligent agent technologies for advancing simulation-based systems engineering via agent-directed simulation. SCS M\&S Magazine, July

20. Zacharewicz G, Frydman C, Giambiasi N (2008) G-DEVS/HLA environment for distributed simulations of workflows. Simulation 84(5):197-213

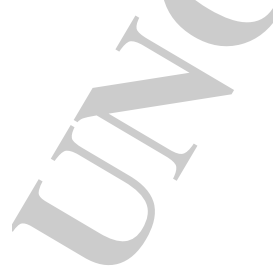

